ISSN: 1130-2887 - eISSN: 2340-4396

DOI: https://doi.org/10.14201/alh.25124

\title{
PLURINACIONALISMO, PLURICULTURALIDAD Y FEDERALISMO EN MÉXICO
}

\section{Plurinationalism, Pluriculturality and Federalism in Mexico}

\author{
Jorge CHAIRES (1) jorge.chaires@academicos.udg.mx ${ }^{1}$ \\ ${ }^{1}$ Universidad de Guadalajara
}

Envío: 2020-11-19

Aceptado: 2021-06-18

First View: 2021-08-04

Publicación: 2021-08-31

RESUMEN: El propósito del trabajo es analizar la viabilidad del federalismo mexicano desde una perspectiva de la plurinacionalidad y la pluriculturalidad. Se parte de la tesis de que el federalismo resulta apropiado en los países plurinacionales, en tanto que en países pluriculturales se puede tratar a través de otro tipo de estructuras político-constitucionales. Se afirma que en México, al no ser un país plurinacional, el federalismo no es una opción apropiada para resolver los problemas de distribución de competencias.

Palabras clave: federalismo; Estado-nación; nación; cultura; plurinacionalismo; pluriculturalismo

ABSTRACT: The purpose of this paper is to analyze the viability of Mexican federalism from a plurinational and pluricultural perspective. It is based on the thesis that federalism is appropriate in plurinational countries, while in pluricultural countries it can be dealt with through other types of political-constitutional structures. It is argued that since Mexico is not a plurinational country, federalism is not an appropriate option to resolve the problems of distribution of competences.

Keywords: federalism; Nation state; nation; culture; plurinationalism; pluriculturalism 


\section{INTRODUCCIÓN}

En México se ha visto el federalismo como simple técnica constitucional de distribución de competencias, el cual puede ser implementado por cualquier país sin importar su contexto histórico e identidad nacional. Además de que dentro de la academia mexicana existe un consenso en justificar el federalismo por su diversidad cultural, geográfica, económica e, incluso, por la variedad de climas o la extensión territorial.

Sin embargo, la doctrina ha planteado la necesidad de correlacionar diversas características que debe reunir un país a fin de que el federalismo pueda resultar una alternativa. Para Will Kymlicka (1996b), el federalismo sencillamente carece de relevancia para muchos tipos de pluralismo etnocultural, pero es potencialmente relevante para el acomodo del pluralismo multinacional. Juan Linz (1999) es de la opinión de que: «El federalismo será una opción y quizá una necesidad cuando en un Estado hay grupos significativos de población con un sentido de identidad nacional» (p. 21). En el mismo sentido, Gaudreault-DesBiens (2006) estima que el federalismo es notablemente apropiado en aquellos Estados que son plurinacionales, en donde las diferencias abundan, mientras que en los países pluriculturales o multiculturales su diversidad puede resolverse legalmente por medio de otro tipo de estructuras político-constitucionales.

El artículo 2. ${ }^{\circ}$ de la Constitución mexicana establece que la nación tiene una composición pluricultural sustentada originalmente en sus pueblos indígenas, así como en los pueblos y comunidades afromexicanas. ¿Ello significa que, al no reconocerse como un país plurinacional, el federalismo no se puede considerar como apropiado para el país y, por el contrario, al definirse como un país pluricultural, esa diversidad cultural puede ser tratada con otro tipo de estructura político-constitucional?

Que la Constitución disponga que México tiene una composición pluricultural, sustentada originalmente en sus pueblos indígenas y en los pueblos y comunidades afromexicanas, no justifica la adopción del federalismo. En primer lugar, porque no se tomaron en cuenta para la división territorial (no existe el Estado huichol, náhuatl, purépecha, etc.); lo que no implica que no se les reconozcan ciertos derechos. En segundo lugar, porque la Constitución acepta la pluriculturalidad solo a partir de los pueblos y comunidades indígenas y afromexicanas y calla respecto a la población occidentalizada o los no indígenas.

A partir de estas abstracciones argumentativas, el presente trabajo tiene como objeto analizar la correlación que existe entre la diversidad nacional y cultural para que el federalismo sea factible en México. Para ello será necesario analizar el federalismo no solo desde su dimensión normativa y como una técnica constitucional, sino en razón de su practicidad a partir de la diversidad nacional y cultural, pero también de la capacidad de contar con gobiernos locales con instituciones democráticas sólidas.

Cabe precisar que el breve estudio que aquí se presenta no busca el reconocimiento explícito de las comunidades indígenas dentro del sistema federal, ya que por su complejidad rebasa el propósito de este trabajo. Conscientes de la seriedad con la que se debe abordar el tema, sin caer en superficialidades y prejuicios sobre la etnología, el estudio se limita a dejar constancia de que los pueblos originarios no fueron considerados para la configuración del sistema federal y, en menor sentido, los pueblos y comunidades afromexicanas, quienes no tienen el status de originarias. 
El trabajo se divide en tres apartados que siguen una línea de argumentación deductiva. En el primer apartado se hace una aproximación conceptual de nación y cultura, de tal manera que facilite entender y marcar las diferencias entre los países plurinacionales y pluriculturales. En el segundo apartado se hace un análisis jurídico del artículo 2..$^{\circ}$ de la Constitución que define a México como un país pluricultural, de tal manera que permita comprender el impacto de su diversidad cultural en el sistema de distribución de competencias. Y, por último, a manera de conclusión, se analiza la correlación entre plurinacionalidad, pluriculturalidad, a efecto de estar en posibilidad de determinar si el federalismo en México se presenta como viable para resolver los problemas de gobernanza.

\section{PLURINACIONALIDAD}

\section{II.1. El Estado-nación}

El Estado-nación tradicionalmente se asoció prima facie a la evolución natural de una organización poblacional nacional: una nación, un Estado, ya sea que se imagine como un proceso étnico-histórico (teoría primordialista o como un proceso histórico-evolutivo, teoría perennialista). No obstante, este paradigma no ha sido estático, sino que ha sido confrontado por otras corrientes que no solo replantean la génesis del fenómeno nación y su pendón el nacionalismo, sino que niegan que exista una relación indisoluble.

La teoría modernista-constructivista vislumbra a la nación no necesariamente como un proceso natural e histórico, sino como una construcción independiente y contingente, incluso como comunidades imaginadas o inventadas, en palabras de Benedict Anderson (1993) y Eric Hobsbawm (1998). Desde esta perspectiva, se pretendió que la unión de pueblos o naciones conformaran un Estado-nación, que apostaba a la unidad para hacer frente no solo a las amenazas externas, sino también internas. El Estado-nación intentó acabar con las diferencias, partiendo de la aceptación de un poder único, la uniformidad legislativa y administrativa, así como la creación de una nueva identidad nacional válida para todos; es decir, una cultura común.

Será la Revolución francesa el parteaguas que abriría el horizonte a la construcción de una nueva relación entre la sociedad y el poder. Según Habermas (2008) el Estado-nación «creó la base para la homogeneidad cultural y étnica, sobre la que desde fines del siglo XVIII pudo ponerse en marcha la democratización del Estado, si bien a costa de la represión y exclusión de minorías nacionales» (p. 621). Sin embargo, la construcción del Estado-nación en muchos casos no logró borrar la diversidad histórica y cultural de los pueblos, lo que ha dado pie a su derrumbe y al resurgimiento de nacionalismos históricamente reprimidos. Algunos grupos del nuevo Estado no se sintieron identificados con la nueva configuración. Para Hastings (2000), esa supuesta unidad cultural y política del Estado era un simple sueño más que una realidad. Afirma que la mayoría de los Estado-nación incluyen grupos de personas que no pertenecen a la cultura original o no se sienten parte de determinada nación, pero que otras, como Inglaterra, ya presentaban características nacionales en la etapa anterior a la Ilustración.

Cuando una pluralidad de pueblos o naciones han sido forzados a subsumirse dentro del Estado-nación, el sentimiento nacionalista no necesariamente se desvanece, sino que sigue 
latente y, por lo general, resurge de manera más vehemente. Se reconoció que dentro de un Estado no siempre existe un único pueblo o una única nación, sino una pluralidad de pueblos y naciones, conformados con distintas culturas, tradiciones y costumbres, incluso con distintos idiomas, religiones y razas (Heller, 1995).

En muchos países el Estado-nación dejó de ser monolítico para reconocerse más bien como un Estado-plurinacional. Ello conduce a preguntar: ¿cuándo se justifica la realización de un pacto o alianza federalista para que diversas naciones o sociedades formen un Estado? La respuesta no es clara debido al carácter polisémico de los conceptos de nación y cultura, que aceptan diferentes interpretaciones.

\section{II.2. El Estado plurinacional}

En virtud de que el Estado plurinacional implica la coexistencia de dos o más pueblos o naciones dentro de un mismo Estado, ello requiere acercarse a un concepto de nación que permita distinguir entre los países plurinacionales y los países pluriculturales y, de esta forma, entender su correlación con los sistemas federales. Cabe advertir, como lo resaltó Ernest Gellner (2001), que las definiciones de plurinacionalidad y pluriculturalidad están supeditadas a dos términos todavía no definidos: nación y cultura.

El concepto de nación tradicionalmente se ha entendido desde puntos de vista étnicoculturales, donde el rasgo característico es la procedencia de ciertos elementos comunes (raza, lengua, religión o un pasado histórico), o desde un enfoque estatista, en donde el elemento político es la característica principal (Touraine, 2000). También se ha identificado desde enfoques objetivos y subjetivos; los primeros estarían relacionados con cuestiones étnico-culturales, en tanto que los segundos se distinguen por la voluntad o conciencia de contar con recuerdos comunes, así como proyectos de futuro compartido, sentimientos de pertenencia al grupo o la voluntad de vivir juntos que se relacionaba con una psicología colectiva.

Sin embargo, estas explicaciones no han sido ajenas a polémicas, ya que resulta imposible delimitar de forma clara y objetiva los grupos humanos definidos por distintos rasgos étnicos o precisar el grado de cohesión política dentro de un Estado de tal manera que no sea artificiosa. Ernest Renan (1987), en su célebre conferencia pronunciada en 1882, titulada ¿Qué es una Nación?, advertía sobre la dificultad de definir el concepto de nación: «Procuremos llegar a alguna precisión en estas cuestiones difíciles donde la menor confusión sobre el sentido de la palabra al inicio del razonamiento puede producir al final los más funestos errores» (p. 60). Renan hizo ver lo discutible que podía ser cada uno de los elementos con los que se quería identificar a la nación (etnográfico, racial, lengua, religión y geografía). Para intentar zanjar la cuestión, aportaría un nuevo elemento al concepto de nación que iba más allá de los rasgos tradicionales y que ha determinado el debate hasta nuestros días: la nación como alma espiritual, traducida en la voluntad de vivir juntos que se materializa a través de un plebiscito cotidiano.

Eric Hobsbawm (1998) concibe la nación como una novedad histórica resultado de la evolución y contexto histórico, pues, para él, la misma palabra ha significado cosas muy diferentes de manera simultánea, por lo que recomienda tomar una postura agnóstica. Hobsbawm (1998) no hace suya ninguna definición apriorística, sino que se limita a dar una definición como supuesto inicial para poder entrar a su estudio: «Se tratará como nación a 
cualquier conjunto de personas suficientemente nutrido cuyos miembros consideren que pertenecen a una nación» (pp. 16-17).

En el mismo sentido, y ante la dificultad de encontrar una definición libre de cualquier tendencia doctrinal, Anthony Smith (2009) propone definir la nación de una manera ideal, advirtiendo que es inevitablemente estipulativa: «Una comunidad humana con nombre y autodefinida cuyos miembros cultivan recuerdos, símbolos, mitos, tradiciones y valores compartidos, habitan y están unidos a territorios históricos o 'patrias', crean y difunden una cultura pública distintiva y observan unas costumbres comunes y leyes estandarizadas» (pp. 29-30).

Para Kymlicka (1996a), la nación significa «una comunidad histórica, más o menos completa institucionalmente, que ocupa un territorio o una tierra natal determinada y que comparte una lengua y una cultura diferenciadas» (p. 26). Kymlicka (1996a) distingue dos modelos amplios de diversidad cultural: el primero que se da con la incorporación de culturas que disfrutaban previamente de autogobierno concentradas en un Estado mayor, las cuales denomina minorías nacionales. Se caracterizan por que a pesar de formar parte de una cultura mayoritaria desean seguir siendo sociedades distintas, por lo que reclaman diversas formas de autogobierno o autonomía. La segunda, como resultado de la inmigración tanto individual como familiar, que las denomina grupos étnicos, los cuales no solo desean integrarse a la sociedad que forman parte, sino también que se les reconozca como miembros de pleno derecho. Se caracterizan por que, si bien desean que se les reconozca su identidad étnica, no pretenden llegar a convertirse en una nación separada y autogobernada.

Para David Miller (1997), la fragmentación cultural de los Estados modernos ha llevado a que exista menos convergencia o acuerdo que nunca. Su composición heterogénea con identidades personales se pone de manifiesto en expresiones como las filiaciones y lealtades étnicas y religiosas. Pero también en puntos de vista sobre la moralidad, gustos artísticos o musicales o lo que se considera valioso e importante.

Esta heterogeneidad de la sociedad no siempre está perfectamente delimitada, ya que, mientras una persona se reconoce como parte de una población originaria mayoritaria, profesa la religión minoritaria. Así como una persona miembro de una minoría racial que decide hablar el lenguaje de la mayoría de la población, como muchos mexicanos que viven en los Estados Unidos que decidieron dejar de hablar castellano.

De igual manera, el aspecto subjetivo de sentirse o querer ser parte de una comunidad y el deseo de vivir juntos, independientemente de que desciendan de una misma raza, hablen el mismo idioma, profesen la misma religión o tengan una historia común, constituyen elementos determinantes, aunque pueden no ser únicos.

Hispanoamérica se independizó de España, en gran medida, por las desigualdades que se fueron creando entre españoles peninsulares y españoles americanos. No obstante que procedían de una misma raza, hablaban un mismo idioma, profesaban la misma religión y se regían por la misma estructura político-administrativa y jurídica, las diferencias se fueron acentuando al grado de no sentirse parte de una misma nación. La geografía, la discriminación, una historia ya no tan común y el deseo de no vivir juntos fueron factores decisivos. De acuerdo con Luis Villoro (2010), la situación inasequible y lejana precipitó la independencia. Asevera que las quejas de los novohispanos eran las mismas: «Falta de empleo, carencia de ilustración, la superioridad con que los mira, el monopolio del comercio y las trabas a la industria» (p. 32). 
Los habitantes de Texas provenientes de los territorios del norte de América decidieron separarse de México, motivados por el hecho de no sentirse ni querer ser parte de la comunidad mexicana (Kökény, 2011, p. 21). Había pocos elementos identitarios en común que les permitieran seguir unidos como nación. No contaban con una historia común, provenían de razas distintas, hablaban otro idioma, no profesaban la misma religión, además de que venían de una estructura política institucional muy diferente.

En tal sentido, determinar si un Estado es plurinacional o no dependerá de muchos factores que influyen todos entre sí, mas no necesariamente tendrían que cumplirse todos, es decir, la raza, la religión, la lengua, las tradiciones o una historia común son condiciones todas ellas importantes, pero no determinantes.

\section{PLURICULTURALIDAD}

La plurinacionalidad de un Estado no se puede confundir con el pluralismo cultural, ya sea que se vea como pluricultural o multicultural. Un país plurinacional es por esencia pluricultural y multicultural, pero un país con diversidad cultural no es necesariamente plurinacional.

Distinguir la diversidad cultural de un país resulta esencial para la construcción del andamiaje constitucional de reparto de competencias no solo como reconocimiento de distintas expresiones culturales, sino también para viabilizar la integración institucional dentro de la diversidad.

Para entender la diversidad cultural del Estado y sus alcances en los sistemas federales, es necesario aproximarnos a una definición de cultura. Sin embargo, es necesario advertir que, al igual que con el concepto de nación, tratar de definir a un país como pluricultural o multicultural es una tarea delicada, en razón de que la misma definición de cultura no solo ha cambiado con el tiempo, sino que existen distintas acepciones.

La diversidad cultural comúnmente suele confundirse entre pluriculturalidad, multiculturalidad o interculturalidad o con términos como plurinacionalidad y plurietnicidad. Ferran Requejo (1996) advierte sobre los riesgos de confundir estos términos en las democracias actuales, ya que bajo el concepto de multiculturalismo se subsumen fenómenos muy distintos entre sí, lo cual implica que se estudien teórica y empíricamente de manera distinta.

Para efectos del presente análisis se utilizan definiciones generalmente aceptadas que, al igual que el concepto de nación, facilitarán la distinción entre un país plurinacional y pluricultural.

Una de ellas es la de Edward B. Tylor (1975), quien entiende la cultura como «todo complejo que incluye el conocimiento, las creencias, el arte, la moral, el derecho, las costumbres y cualesquiera otros hábitos y capacidades adquiridas por el hombre en cuanto miembro de la sociedad» (p. 29). Kymlicka (1996a) prefiere referirse a una cultura societal: «Una cultura que proporciona a sus miembros unas formas de vida significativas a través de todo el abanico de actividades humanas, incluyendo la vida social, educativa, religiosa, recreativa y económica» (p. 112). Estas sociedades, según Kymlicka, comparten no solo un territorio, sino también una lengua o léxico cotidiano de la vida social; en términos de Charles Taylor (2009), un 
lenguaje del arte, del gesto, del amor y similares, que solo se aprenden mediante intercambio con los demás.

En tal sentido, se puede decir que la diversidad cultural y la nacional se distinguen por su grado. La cultura no se edifica en raíces étnicas distintivas, aunque no las niega o desconoce, sino que son tomadas en cuenta para contrastar las diferencias culturales tanto en el ámbito individual como en el comunitario. Mientras que en la nación están involucradas cuestiones de etnicidad como pueden ser la raza, la religión, la lengua, un pasado y un futuro comunes, la cultura se relaciona con ciertos valores, tradiciones, creencias y modos de vida, con la peculiaridad de que son adquiridos o asimilados.

Ahora bien, a efectos de sistematizar las innumerables definiciones sobre pluriculturalidad, multiculturalidad e interculturalidad, el presente análisis se apoya en los trabajos de María del Mar Bernabé e Isabel Wences.

María del Mar Bernabé (2012) explica que por pluriculturalidad se entienden las diversas culturas que conviven en un mismo territorio, es decir, la presencia simultánea de dos o más tendencias ideológicas y grupos sociales que interactúan en una unidad estatal. En cuanto a la multiculturalidad, aunque también el prefijo «multi» hace referencia a una cierta cantidad, esta se debe entender como la presencia de diferentes culturas en un mismo territorio, que se limitan a coexistir, pero no a convivir. Se caracteriza por que las diferentes culturas no interactúan necesariamente, sino que prevalece un escenario de segregación y de negación de la convivencia. En tanto que interculturalidad, el mismo prefijo lo define como «entre culturas», que lleva al entendimiento «entre» las diferentes culturas que habitan un mismo territorio.

Para Isabel Wences (2015) el interculturalismo se entiende como un modelo de diversidad cultural en donde los factores esenciales serán la práctica de integración, así como el rechazo a toda discriminación y la promoción de una cultura pública.

En México se congregan las diferentes expresiones culturales, es decir, se pueden apreciar manifestaciones de pluriculturalidad como multiculturalidad e interculturalidad. Llama la atención que la relación con las comunidades indígenas es de coexistencia y no de convivencia, lo cual se ha querido negar jurídicamente.

En México existe una identidad nacional occidental por encima de las identidades culturales locales también occidentales, la cual se caracteriza por el idioma, la religión, la raza mestiza y una estructura jurídico-institucional. De acuerdo con José del Val (2004), la identidad nacional durante el siglo XX estuvo cimentada en tres elementos: aztequismo, guadalupanismo y un partido político hegemónico. Raúl Béjar y Héctor Rosales (1999) se refieren a cinco categorías básicas que desde la antropología se han elaborado: indianismo, occidentalismo, mestizaje, yuxtaposición e hibridismo.

Frente a esta identidad nacional aparecen las identidades locales (no étnicas) que se concretan en diferentes expresiones culturales que no van más allá de simples costumbres o estilos de vida. Son defendidas como parte de su identidad local que los distingue de otras regiones: «Nosotros es nuestra identidad; ellos son las identidades diferentes que determina la nuestra» (Sartori, 2001, p. 48). 


\section{FEDERALISMO Y LA PLURICULTURALIDAD DEL ESTADO MEXICANO}

\section{IV.1. La disyuntiva federalista}

Siguiendo esta línea deductiva conceptual está claro que México no entra en la concepción de países plurinacionales. Juan Linz (1999) enmarca dentro de los países mononacionales o naciones-Estados a los países surgidos de procesos independentistas en Latinoamérica, como Argentina, Brasil, México y Venezuela. Estos países, precisa Linz, se decidieron por el federalismo, cuya finalidad era mantener unidas las partes heterogéneas de un Estado ya existente.

Hay dos características que se estima que son sustanciales para que un país se decante por el sistema federal: por un lado, el autogobierno de las partes constitutivas y, por otro lado, la diversidad nacional y cultural, en la que se centra este trabajo.

$\mathrm{El}$ federalismo, de acuerdo con la doctrina, tiene su origen en un sistema de reparto de competencias sustentado en la cosoberanía que, según Michael Burgess (2006), se explica debido a una experiencia política de autogobierno de muchos años. Para Robert Friedlander (1979), la autorregulación y el autogobierno de las colonias inglesas alimentaron sentimientos de identidad y pertenencia que sembrarían las semillas del federalismo. Daniel Elazar (1987) asevera que los sistemas federales exitosos parecen requerir una base cultural apropiada, que entrelaza el autogobierno y el gobierno compartido.

El federalismo en México no se ajusta a la idea de cosoberanías, porque las partes constitutivas no gozaban de una cultura política federal, de tal manera que permitan hablar de verdaderos entes autogobernables. Al momento de la independencia las diferentes provincias de la Nueva España no estaban preparadas para asumir la gran responsabilidad de tomar las riendas de su gobierno. José Carlos Chiaramonte (2016) atribuye el fracaso del federalismo en los países latinoamericanos a la debilidad de las provincias, que, según el historiador argentino, si se las compara con los Estados norteamericanos en el momento de la independencia, la mayoría poseía menor solidez institucional y menor poderío económico.

Por lo que tiene que ver con la segunda característica, en México se defiende el federalismo por su diversidad cultural (no étnica), lo que justificaría que en la Constitución se hable de estados libres y soberanos en todo lo concerniente a su régimen interior. Juristas y politólogos insisten en resaltar esas diferencias para justificar el modelo de distribución de la competencial federal.

Según el jurista mexicano Jacinto Faya Viesca (2014), el federalismo parte de una unidad y diversidad que lo caracteriza. Y que así debe entenderse, en razón de que es «una nación con una gran heterogeneidad en lo económico, en lo cultural y en la distribución de la riqueza, según sea la región o entidad federativa» (p. 2). Por su parte, Ricardo Raphael (2016) defiende el federalismo: «Por su geografía, biología y demografía el país nació plural. De ahí sus múltiples comunidades, culturas e identidades. Cada vez que olvidamos ese origen violentamos los cimientos de la República» (párr. 3).

No obstante, como se expuso en la parte introductoria, el federalismo, de acuerdo con autores como Will Kymlicka (1996b), no constituye una respuesta apropiada a las diferentes formas de pluralismo cultural, pero sí puede ser relevante para el acomodo del pluralismo multinacional. Para Linz (1999), el federalismo resulta una opción en aquellos Estados en donde existen poblaciones con un sentido de identidad nacional. En el mismo sentido, 
Jean-François Gaudreault-Desbiens (2006) estima que el federalismo resulta notablemente apropiado en aquellos Estados que son plurinacionales, en donde las diferencias abundan entre los estados, mientras que en los países pluriculturales o multiculturales su diversidad se puede gestionar a través de otro tipo de estructuras político-constitucionales.

En tal sentido, resulta importante saber a qué tipo de diversidad cultural se refieren los que defienden el federalismo en México. La Constitución, en este sentido, no nos resuelve el problema, ya que la pluriculturalidad se reconoce en función de los pueblos y comunidades indígenas y afromexicanas. El primer párrafo del artículo 2. ${ }^{\circ}$ dispone que: «La Nación tiene una composición pluricultural sustentada originalmente en sus pueblos indígenas». En tanto que, en su inciso $C$, se señala que: «Esta Constitución reconoce a los pueblos y comunidades afromexicanas, cualquiera que sea su autodenominación, como parte de la composición pluricultural de la Nación».

El rango constitucional de los pueblos y comunidades afromexicanas implicó el reconocimiento de los mismos derechos políticos y jurídicos de los pueblos y comunidades indígenas, a fin de garantizar su libre determinación, autonomía, desarrollo e inclusión social, pero sin que se puedan considerar como originarias para efectos de la división territorial y competencial.

No se puede hablar de la diversidad étnica como sustento del federalismo porque los pueblos indígenas $\mathrm{y}$, mucho menos, los pueblos o comunidades afromexicanas no fueron parte del pacto federal. Como lo dice Luis Villoro (1998), el federalismo en México no correspondió a la diversidad real de los pueblos, sino que fue el resultado de intereses locales o para intentar dar solución a conflictos de poder. Aclara que no fue consecuencia de Estados independientes y soberanos que defendieran sus usos y costumbres. Precisa que: «El federalismo es una expresión más del ideal de una patria unida, constituida no por culturas diversas, sino por individuos iguales entre sí» (pp. 42 -43).

La división territorial bajo el modelo federal no sirvió para reconocer a las diferentes etnias existentes, sino que, por el contrario, las ignoró (López, 2016). El estado de Oaxaca no es libre y soberano por los usos y costumbres de sus comunidades indígenas. Chihuahua no es libre y soberano porque habitan en su territorio los tarahumaras. Los territorios ancestrales fueron divididos arbitrariamente entre varios estados. Existen comunidades huicholas en los estados de Nayarit, Jalisco, Zacatecas y Durango. Los purépechas habitan primordialmente en los estados de Michoacán, Estado de México, Guanajuato y Oaxaca. Los mazahuas quedaron asentados dentro de los estados de Michoacán y Estado de México. Los mixtecos en Guerrero, Oaxaca y Puebla. Los mayas en Yucatán, Campeche y Quintana Roo, incluso en Belice y Guatemala. En tal sentido, resulta cuestionable el discurso de quienes apelan a la existencia de la diversidad étnica del país como justificación del federalismo.

Cabe destacar que la redacción del artículo $2 .^{\circ}$ de la Constitución fue incorporada en el 2001, como consecuencia del movimiento indígena que estalló en enero de 1994, por lo que no está relacionado con la adopción del federalismo. En tanto que el reconocimiento de los pueblos y comunidades afromexicanas se incorporó al texto constitucional en el 2019, después de que su invisibilidad fue denunciada como violación a diversos pactos y tratados internacionales, como el Pacto Internacional de Derechos Civiles y Políticos y la Convención Americana sobre Derechos Humanos Pacto de San José de Costa Rica (Molina, 2020). 
El rango constitucional también implicó la distinción jurídica entre pueblos y comunidades indígenas. El artículo 2. ${ }^{\circ}$ señala que las comunidades son las que integran un pueblo indígena; que formen una unidad social, económica y cultural; asentadas en un territorio, y que reconocen autoridades propias de acuerdo con sus usos y costumbres. Esta distinción resulta relevante en el ámbito constitucional, ya que las comunidades no solo tienen derecho a que se les reconozcan jurídicamente sus usos y costumbres, sino también el pleno derecho de acceso a la justicia constitucional a través de los derechos colectivos.

Ahora bien, en una visión federalista el constituyente dispuso que serían las entidades federativas las que determinarían las características de autonomía y libre determinación que mejor expresasen las situaciones y aspiraciones de sus pueblos indígenas. No obstante, la descentralización se ha enfrentado a disyuntivas que no han encontrado soluciones satisfactorias.

En primer lugar, el que la Constitución estableciera ciertos lineamientos y parámetros acotó el margen de actuación de las legislaturas locales, lo cual se acerca más a las reglas de un federalismo simétrico. Si bien es cierto que en los gobiernos locales se aprecian distintas fórmulas legales e institucionales, así como el reconocimiento de ciertos derechos, estos no pueden ir más allá de lo prescrito en el texto constitucional y de las disposiciones de carácter internacional. Los tribunales electorales tienen la posibilidad de validar o invalidar procedimientos y mecanismos tradicionales indígenas en caso de que se aparten del texto constitucional o de los estándares internacionales (Jiménez y Aguilar, 2013).

En segundo lugar, la fragmentación legislativa derivó a que cada estado adoptase las nuevas tendencias y avances del derecho a diferentes ritmos y cadencias, lo que se conoce como la brecha de implementación. Mientras que en algunos estados se aprueban fórmulas novedosas, otros estados no cuentan con una ley de derechos indígenas, lo que evidencia que los términos y alcances del reconocimiento de derechos varía considerablemente (Wright et al., 2019, p. 68).

La falta de armonización en materia de derechos indígenas se ha visto como una situación grave, que pudiese derivar en un contexto de desigualdades y discriminación en términos jurídicos. Así lo advierte Francisco López Bárcenas (2010), para quien lo peor de la remisión legislativa a los estados es que «estando varios pueblos asentados en diferentes estados, mientras todos no legislen de igual manera, los derechos de un mismo pueblo indígena no serán reconocidos de igual forma dando lugar a la discriminación que se buscaba evitan» (p. 58). Frente a ello los tribunales han asumido un rol esencial, ajustando sus criterios a estándares internacionales, al grado de que se ha señalado que la ausencia de una ley no es argumento suficiente para no reconocer sus derechos.

\section{IV.2. La pluriculturalidad del Estado mexicano de acuerdo con la Constitución}

Al disponer la Constitución que la nación mexicana tiene una composición pluricultural sustentada originalmente en sus pueblos indígenas, así como en los pueblos y comunidades afromexicanas, nos lleva a hacer varias reflexiones:

Primero, reconocer la pluriculturalidad a partir de los pueblos y comunidades indígenas y afromexicanas implica que existe una población que no lo es, que se asume como culturalmente homogénea. La Constitución reconoce la pluriculturalidad de la nación mexicana a 
partir de sus pueblos originarios y afromexicanos, pero calla respecto a la composición de los que no lo son, lo cual no significa que la población no indígena se excluya de la Constitución. En este sentido y bajo una interpretación normativa declarativa productora de otra norma, se asume que si existe una parte es porque hay por lo menos otra que la complementa, es decir, la población no originaria. ¿Y esa población qué es? Lo que parece una obviedad resulta ser la reafirmación de una comunidad indígena y afromexicana y otra no indígena.

Segundo, el constituyente redujo las diferencias entre las comunidades indígenas y afromexicanas y las que no lo son a simples cuestiones culturales. Al decir que la nación tiene una composición pluricultural sustentada originalmente en sus pueblos indígenas, así como en los pueblos y comunidades afromexicanas, se debe entender que las diferencias entre ellos y quienes no lo son se reducen a simples cuestiones culturales. Es decir, las distintas razas, dialectos, cultos religiosos y el pasado histórico de los distintos pueblos originarios y afromexicanos son percibidos como simples diferencias culturales.

Los estudiosos en el tema indígena refieren que las diferencias entre unos y otros no son solo culturales, sino de identidad étnica o nacional. Las diferencias entre las comunidades étnicas y el mundo occidentalizado no se reducen a cuestiones de raza, religión, pasado histórico o un sistema propio de orden social, sino también implican una cosmovisión muy distinta, que determina la forma de ver y entender la vida (Bello, 2004). Lo que ha llevado a que consideren a las comunidades indígenas como naciones atrapadas, naciones potenciales o nación en ciernes (nation to be) (Smith, 1997, p. 69).

Tercero, la Constitución colocó en un plano de igualdad cultural a los mexicanos no originarios. Al no hacerse ninguna precisión en la Constitución respecto de la composición de la población occidentalizada o no indígena, se deduce que los ubica en un plano de igualdad cultural. De acuerdo con las definiciones de cultura, es claro que existen muchas diferencias culturales entre las regiones occidentalizadas del país o no indígenas, pero solo eso, rasgos distintivos culturales. Las diferencias no van más allá de modos de vida, costumbres, tradiciones o grados de desarrollo ya sea industrial, artístico o científico, que bien pueden enmarcarse en lo que se ha llamado ciudadanía diferenciada. Pero que de ninguna manera llegan a separarse en cuanto a identidades nacionales que involucren raza, lengua, religión, sistemas e instituciones de gobierno o un pasado y un futuro en común.

Lo anterior no implica que se esté en favor de un rediseño de distribución territorial y competencial en función del reconocimiento de una plurinacionalidad indígena y afromexicana y otra occidental, porque la integración es la esencia de la identidad nacional mexicana que se complementa y la enriquece. En todo caso, deberán ser las comunidades indígenas las que decidan el sistema de distribución de competencias que mejor les convenga. El planteamiento es respecto a un sistema competencial en donde la gran mayoría de la población no es indígena ni afromexicana.

De acuerdo con el censo de población 2020, la población en México es de poco más de 126 millones de habitantes. Aproximadamente el $21.5 \%$ del total de la población se autorreconoce indígena, aunque solo 6 de cada 100 habitantes en México habla alguna lengua nativa, es decir, 7.3 millones de personas (en 1930 había 16 millones); y, de ellas, poco más de 900.000 no hablan el castellano y solo se comunican en su lengua. La población que se reconoce como afrodescendiente es de 2.5 millones, que representa el $2 \%$ de la población total (INEGI, 2020). 
La población indígena y afromexicana se encuentra dispersa en todo el país. La mayoría de la población indígena se concentra en seis estados (seis de cada diez residen en los estados de Oaxaca, Chiapas, Puebla, Estado de México, Veracruz y Yucatán); en los estados de Aguascalientes, Colima, Zacatecas y Coahuila, representa menos del $1 \%$. Algunos pueblos originarios están concentrados en un territorio muy pequeño, mientras otros están asentados entre varios estados. Existen comunidades conformadas por hasta un millón y medio de personas de habla náhuatl y otras en las que solo viven pocas personas que hablan una sola lengua indígena (20, awakateko; 63, kickapoo; 71 , ayapaneco) ${ }^{1}$. En tanto que la población afromexicana se concentra principalmente en los estados de Guerrero, Oaxaca y Veracruz.

La gran mayoría de los mexicanos se puede asumir de origen mestizo, además de que poco más de 84 millones de mexicanos profesan la religión católica. Los 120 millones de mexicanos, salvo las excepciones y peculiaridades que reconoce la Constitución en favor de las comunidades indígenas, se rigen bajo el mismo sistema político-jurídico.

Los rasgos distintivos de la cultura occidentalizada en el país no llegan a ser tan fuertes que conduzcan a asociarlos con los atributos distintivos de la nación. Que una región del país tenga determinados modos de vida, tradiciones, costumbres, conocimientos o niveles de desarrollo, distintos a otras regiones, no significa que se esté en presencia de diferentes identidades nacionales que justifiquen el federalismo.

En consecuencia, la realidad es que México es un Estado-nación o mononacional, para utilizar el término de Juan Linz (1997), con una diversidad cultural pluricultural, multicultural e intercultural. Conformada por una población mayoritariamente occidentalizada, de origen mestizo, que habla el mismo idioma, profesa mayoritariamente la misma religión, regida por un mismo sistema político y jurídico, con un pasado común y, hasta hoy, con la voluntad de vivir juntos. Con una minoría que se identifica como indígena (21.5\%), aunque solo el $6.5 \%$ habla alguna lengua nativa distinta al castellano, y otra que se identifica como descendiente africana que representa el $2 \%$ de la población total.

\section{LA DIVERSIDAD NACIONAL COMO ELEMENTO DETERMINANTE PARA EL FEDERALISMO. A MANERA DE CONCLUSIÓN}

Como se indicó en el desarrollo del trabajo, para algunos autores el federalismo no constituye una respuesta apropiada a las diferentes formas de pluralismo cultural, pero puede ser relevante para el acomodo del pluralismo multinacional (Kymlicka, 1996b; GaudreaultDesBiens, 2006; Linz, 1999).

Ello deja la interrogante de cuándo se está en presencia de un país plurinacional y cuándo en presencia de un país pluricultural, que justifique la implementación del sistema federal. Para llegar a determinar la viabilidad del sistema federal en México, es imprescindible saber qué es; es decir, si países como México se pueden identificar como países plurinacionales o pluriculturales.

1. Datos al 2020: http://cuentame.inegi.org.mx/hipertexto/todas_lenguas.htm. 
Algunos países han encontrado en la fórmula federalista la solución a los problemasnecesidades de unidad, a partir del reconocimiento de su composición plurinacional, no sin grandes dificultades. Hay que aclarar que no es necesario conceptualizar un país como plurinacional para decantarse por el sistema federal, pues, como ya vimos, a partir de los problemas para definir una nación y, por la misma razón, un país plurinacional, cada Estado adoptará el sistema de reparto de competencias de acuerdo a sus muy particulares características y necesidades. Como bien lo advierte Bartolomé Clavero (2015): «Tenemos casos de federalismo no plurinacional y de plurinacionalidad no federal junto a singularidades ni federales ni plurinacionales como la española fallida» (párr. 9).

Países como Bolivia y Ecuador se han proclamado constitucionalmente como países plurinacionales por su diversidad indígena, pero sin adoptar el modelo federal. De acuerdo con el artículo $1 .^{\circ}$ de su Constitución, Bolivia se define como «Estado Unitario Social de Derecho Plurinacional Comunitario». No obstante, para Donna Lee Van Cott (2005, p. 25), la representatividad de los grupos indígenas puede tener un mayor éxito en países descentralizados que en países centralizados, debido a la oportunidad de desarrollarse en niveles geográficos pequeños, al grado de constituir partidos políticos indígenas. En estos casos el federalismo se presenta como opción a la distribución territorial y competencial, a partir del reconocimiento de una verdadera autonomía y libre determinación de los pueblos indígenas.

Ahora bien, si no es necesario identificar a un país como plurinacional para concluir que el mejor sistema para resolver su reparto competencial es el federalismo, sí es importante que las diferencias sean significativas, que justifiquen la celebración de pacto o alianza entre distintas sociedades a fin de defender sus identidades, con la consecuente pérdida de su soberanía. Para Requejo (1996), solo en aquellas reivindicaciones nacionalistas e indígenas resulta relevante la discusión sobre el federalismo como un posible modelo de articulación política, ya que están involucrados criterios de territorialidad y autogobierno.

La Constitución mexicana prescribe que la nación tiene una composición pluricultural sustentada originalmente en sus pueblos indígenas, así como en los pueblos y comunidades afromexicanas. Esta definición jurídico-constitucional de lo que es México constriñe, por un lado, a las comunidades indígenas y afromexicanas a simples entes culturales, aunque con el reconocimiento de ciertos derechos políticos y jurídicos. Por otro lado, acepta que las diferencias entre quienes se reconocen como originarios, afromexicanos u occidentalizados son simplemente de índole cultural, es decir que no son diferencias nacionales.

En México no existe un debate sobre su identidad nacional en razón de que se asume como un hecho irrefutable que México es un país federal por su pluriculturalidad. De acuerdo con José del Val (2004), la ausencia de análisis acerca de la identidad nacional elaborada por los mismos mexicanos se debe a la percepción en la que cada investigador se asume como mexicano, lo que ocasiona distorsiones «epistemológicas heisenbergianas» (p. 16).

Afirmar que el federalismo mexicano es el resultado de la pluriculturalidad no nos aclara a qué diversidad cultural se refiere, porque la Constitución precisa que es en función tan solo de sus comunidades originarias.

Un país con una gran extensión territorial dividido caprichosamente en 32 entidades federativas creó de manera artificial regionalismos culturales. Dichas diferencias no conducen a la existencia de identidades nacionales como nuevoleonesa, chiapaneca, hidrocálida, 
oaxaqueña, etc., y mucho menos que se justifique que se les otorgue el status de Estados libres y soberanos. Se debe tener en cuenta, como vimos, que la extensión territorial, la diversidad cultural o la simple existencia de un mandato constitucional no son argumentos suficientes para reconocer a un país como federal (Blanco, 2012), sino que implica una serie de características muy particulares que México no reúne. Un análisis aparte merece las comunidades originarias, que tienen todo el derecho de reivindicar su soberanía y autodeterminación, no ya como comunidades culturales según las reconoce la Constitución, sino como verdaderas naciones, con todo lo que ello pudiese implicar.

Este desconocimiento de la diversidad cultural nacional ha arrastrado a México a un diseño constitucional artificioso, a partir de una concepción tergiversada de lo que es México y lo que es el federalismo. Ha despojado, a la vez, de una capacidad crítica y creativa que permita estructurar un efectivo andamiaje constitucional con los debidos pesos y contrapesos, particularmente en el ámbito local, en donde no se ha logrado consolidar la institucionalidad necesaria para contar con gobiernos fuertes y eficaces.

No se puede perder de vista que el federalismo cobra sentido en aquellos países en donde los dos distintos órdenes de gobierno cuentan con instituciones suficientes y capaces de funcionar en sus áreas de competencias. El federalismo se presenta como alternativa en países con regiones autogobernables, con una fuerte cultura democrática y de respeto al Estado de derecho o, en términos de los clásicos: federal political culture (Elazar, 1987); de lo contrario, se convierten en espacios de dependencia y sumisión, o bien, de autocracia y autarquía.

En México la agenda de las comunidades locales se impone desde lo nacional no solo por una arraigada aspiración centralista, sino también y primordialmente por la incapacidad de los gobiernos subnacionales de autogobernarse y contar con instituciones auténticamente democráticas y efectivas. El subdesarrollo, la corrupción y la impunidad de México no son atribuibles solo al gobierno federal, sino que, en gran medida, han sido responsabilidad de los gobiernos locales, quienes no han sido capaces de articular los mecanismos democráticos para impulsar el desarrollo de sus regiones.

De acuerdo con Pablo González Casanova (1998), gran parte de lo que sucedía al interior de los estados dependía de los gobernadores: «Dueño y señor de todo el territorio y el destino de sus habitantes» (p. 47). Rogelio Hernández Rodríguez (2009) sostiene que el poder de los gobernadores históricamente ha sido considerable, quienes han cometido todo tipo de arbitrariedades y tropelías. Asevera que el Ejecutivo federal solo interviene cuando se ha puesto en riesgo la estabilidad del estado, debido a la evidente anarquía que amenaza a las instituciones.

El Instituto Mexicano para la Competitividad (IMCO), en sus distintas evaluaciones, ha advertido sobre la persistencia de malas prácticas y opacidad en materia de transparencia financiera en las entidades federativas y municipios (IMCO, 2019). La Auditoría Superior de la Federación (ASF) ha evidenciado la gravedad del mal manejo de los recursos públicos por parte de los gobiernos locales, tanto estatales como municipales (ASF, 2013).

Se debe considerar, como lo han señalado diversos especialistas, que los estados federales no son necesariamente más democráticos, sino que existen serias tensiones entre el ideal democrático y el federalismo (Linz, 1999). Incluso se ha señalado que el federalismo no es la panacea ni todo lo arregla (cure-all) (Loveman, 1996, p. 7), ya que los sistemas federales sin 
una cultura ni práctica política de apoyo pueden generar peores resultados que los sistemas centralistas.

En definitiva, la diversidad cultural que existe en México no justifica un federalismo dual con estados libres y soberanos, sino que su pluriculturalidad bien se puede gestionar por medio de otro tipo de estructuras político-constitucionales que procuren un desarrollo equitativo y homogéneo en todo el país.

Ante la incapacidad de los estados para edificar sistemas institucionales sólidos, un federalismo nacional se presenta como una alternativa, como lo han sugerido diversos juristas: Jorge Carpizo (1972), Ignacio Burgoa (1997), Pedro Zorrilla (1994), Leonel Armenta (2010), Serna de la Garza (2003) y Enrique Aguirre (1997). Es decir, un sistema de homologación y unificación de leyes e instituciones, establecidas en la Constitución y reguladas por el Congreso general, de tal manera que se evite caer en la tentación del centralismo por parte del Ejecutivo federal.

Ahora bien, no se puede desdeñar que las distintas realidades norte-sur han despertado sentimientos diferenciadores, focalizados primordialmente en cuestiones de desarrollo económico y social, que les permiten a determinados gobiernos subnacionales hacerle frente al gobierno central. Dichos sentimientos no tienen que ver con cuestiones culturales, porque se asume que es propio de cualquier país federal, sobre todo, en aquellos países con gran extensión territorial. Tampoco se trata de distintas identidades nacionales (raza, lengua, religión, sistemas político-jurídico o histórico) porque, como ya vimos, no existen (salvo las comunidades indígenas), sino que tiene que ver con la aportación al Producto Interno Bruto del país. El norte del país, con un mayor progreso económico, comienza a exigir una distribución fiscal mucho más equitativa, que puede dar pie a una nueva reconfiguración del sistema competencial y del pacto fiscal, a través de un federalismo asimétrico con pactos regionales $\mathrm{o}$ individuales.

\section{BIBLIOGRAFÍA}

Aguirre, E. (1997). Los retos del derecho público en materia de federalismo: hacia la integración del derecho administrativo federal. UNAM.

Anderson, B. (1993). Comunidades imaginadas. Fondo de Cultura Económica.

Armenta, L. (2010). El federalismo mexicano: una ficción politica. UNAM.

ASF (2013). Diagnóstico sobre la opacidad en el gasto federalizado. Cámara de Diputados. https://www.asf. gob.mx/uploads/56_Informes_especiales_de_auditoria/Diagnostico_sobre_la_Opacidad_en_ el_Gasto_Federalizado_version_final.pdf

Bello, Á. (2004). Etnicidad y ciudadanía en América Latina: La acción colectiva de los pueblos indigenas. CEPAL. https://repositorio.cepal.org/bitstream/handle/11362/2394/ S043148_es.pdf?sequence $=1 \&$ isAllowed $=\mathrm{y}$

Béjar, R. y Rosales, H. (Coords.) (1999). La identidad nacional mexicana como problema político y cultural. Siglo Veintiuno.

Bernabé, M. ${ }^{a}$ del M. (2012). Pluriculturalidad, multiculturalidad e interculturalidad, conocimientos necesarios para la labor docente. Hekademos, 1(11), 67-76.

Blanco, R. (2012). Los rostros del federalismo. Alianza.

Burgess, M. (2006). Comparative federalism. Theory and practice. Routledge. 
Burgoa, I. (1997). Derecho constitucional mexicano. Porrúa.

Carpizo, J. (1972). Sistema federal mexicano. Fondo de Cultura Económica.

Chiaramonte, J. C. (2016). Raíces históricas del federalismo latinoamericano. Sudamericana.

Clavero, B. (2015, 14 de enero). ¿A qué estado decimos federal y a cuál plurinacional? Eldiario.es. https://www.eldiario.es/contrapoder/federalismo-plurinacional_132_4422230.html

Del Val, J. (2004). México: identidad y nación. UNAM.

Elazar, D. J. (1987). Exploring federalism. University of Alabama Press.

Faya Viesca, J. (2014). El federalismo mexicano. Porrúa.

Friedlander, R. (1979). Autonomy and the Thirteen Colonies: Was the American Revolution really Necessary. Duquesne Law Review, 18(3), 507-520.

Gaudreault-DesBiens, J. (2006). Federalismo y democracia. Boletín Mexicano de Derecho Comparado, 39(117), 671-691.

Gellner, E. (2001). Naciones y nacionalismo. Alianza.

González, P. (1998). La democracia en México. Ediciones Era.

Habermas, J. (2008). Facticidad y validez. Trotta.

Hastings, A. (2000). La construcción de las nacionalidades: Etnicidad, religión y nacionalismo. Akal.

Heller, H. (1995). La soberania. Contribución a la teoría del derecho estatal y del derecho internacional. Fondo de Cultura Económica.

Hernández, R. (2009). Los gobernadores y el federalismo. La descentralización del poder. En L. Meyer e I. Bizberg (Coords.), Una historia contemporánea de México, Tomo 3, Las Instituciones. Océano-El Colegio de México.

Hobsbawm, E. J. (1998). Naciones y nacionalismo desde 1780. Crítica.

IMCO. (2019). Transformando la transparencia: Índice de Información Presupuestal Estatal 2019. https://imco. org.mx/transformando-la-transparencia-indice-de-informacion-presupuestal-estatal-2019/

INEGI. (2020). Censo de Población y Vivienda 2020. https://www.inegi.org.mx/programas/ccpv/2020/ default.html\#Resultados_generales

Jiménez, L. y Aguilar, M. (2013). Estándares internacionales de los derechos de los pueblos indígenas aplicados a la materia electoral. Comentarios a las Sentencias del Tribunal Electoral del Poder Judicial de la Federación, n. ${ }^{\circ} 53$

Kökény, A. (2011). Extranjeros en la propia patria - Juan N. Seguín: los tejanos y las guerras de independencia de México y Texas. Acta Hispanica, 16, 19-32.

Kymlicka, W. (1996a). Ciudadanía multicultural. Paidós.

Kymlicka, W. (1996b). Federalismo, nacionalismo y multiculturalismo. Revista Internacional de Filosofía Política, 7, 20-54.

Linz, J. J. (1999). Democracia, multinacionalismo y federalismo. Revista Española de Ciencia Politica, 1, $7-40$.

López, F. (2010). Legislación y derechos indígenas en México. El Colegio de San Luis, A. C.

López, F. (2016). Los pueblos indígenas en las constituciones de México. Argumentos, 29(82), 161-180.

Loveman, B. (1996, 5-7 de diciembre). Federalism and democratization in Latin America: A comparative analysis and partial reform agenda. Coloquio II, Instituto Cultural Ludwig von Mises y Comisión Mexicana de Derechos Humanos, Oaxaca, México.

Miller, D. (1997). Ciudadanía y pluralismo. La Política, 3, 69-92.

Molina, N. (2020). Los afromexicanos como minoría étnica y la cuestión de los derechos humanos. Tlamati, 11(1), 27-30.

Raphael, R. (2016, 21 de abril). Federalismo incongruente. El Universal. https://www.eluniversal.com.mx/entrada-de-opinion/columna/ricardo-raphael/nacion/politica/2016/04/21/ federalismo-incongruente 
Renan, E. (1987). ¿Qué es una nación? Cartas a Strauss. Alianza.

Requejo Coll, F. (1996). Pluralismo, democracia y federalismo: Una revisión de la ciudadanía democrática en estados plurinacionales. UNED.

Sartori, G. (2001). La sociedad multiétnica. Taurus.

Serna de la Garza, J. M. (Ed.) (2002). Federalismo y sistemas de distribución de competencias legislativas. UNAM.

Smith, A. D. (1997). La identidad nacional. Trama.

Smith, A. D. (2009). Ethno-symbolism and nationalism: A cultural approach. Routledge.

Taylor, C. (2009). El multiculturalismo y «la política del reconocimiento». Porrúa.

Touraine, A. (2000). ¿Podremos vivir juntos?. Fondo de Cultura Económica.

Tylor, E. B. (1871). La ciencia de la cultura. JS Kahn (1975). El concepto de cultura. Textos Fundamentales, pp. 29-46.

Van Cott, D. L. (2005). From movements to parties in Latin America: The evolution of ethnic politics. Cambridge University Press.

Villoro, L. (1998). Estado plural, pluralidad de culturas. UNAM-Paidós.

Villoro, L. (2010). El proceso ideológico de la Revolución de independencia. Fondo de Cultura Económica.

Wences, I. (2015). Diversidad cultural y acomodos razonables. Una lectura desde la no dominación. Foro Interno, 15, 39-67. https://doi.org/10.5209/rev_FOIN.2015.v15.50378

Wright, C.; Aguirre, N. y Rodríguez, L. A. (2018). El derecho a la consulta en materia electoral de los pueblos y las comunidades indígenas. Politica Electoral Incluyente, n. ${ }^{\circ}$ 5. Toluca, México. https:// publicaciones.ieem.org.mx/index.php/publicacionesieem/catalog/view/17/11/74-1

Zorrilla, P. (1994). Descentralización politica. UNAM. 
